\title{
Criminologie
}

\section{L'hégémonie et les vocabulaires de la motivation punitive : la gestion discursive des crises sociales}

\section{Dario Melossi}

Volume 25, numéro 2, 1992

Nouvelles connaissances et nouvelles questions en criminologie

URI : https://id.erudit.org/iderudit/017324ar

DOI : https://doi.org/10.7202/017324ar

Aller au sommaire du numéro

Éditeur(s)

Les Presses de l'Université de Montréal

ISSN

0316-0041 (imprimé)

1492-1367 (numérique)

Découvrir la revue

Citer cet article

Melossi, D. (1992). L'hégémonie et les vocabulaires de la motivation punitive : la gestion discursive des crises sociales. Criminologie, 25(2), 93-114.

https://doi.org/10.7202/017324ar
Résumé de l'article

In this paper, the author argues that, "in a given society, a process of increasing carceration occurs in cunjonction with situations perceived as critical for the maintenance of the elites' hegemony in that society (irrespective of "crimes rates"). He revisits the rich tradition inspired by Rusche's original hypothesis, but avoids the economist and instrumentalist temptations of that work. Evidence for the linkage between social structure and punishment is to be found in what he calls "vocabularies of punitive motive". He also shows that this theoretical reconstruction dovetails well with the crucial work of Foucault and other contributions to the sociology of law. 
In this paper, the author argues that, "in a given society, a process of increasing carceration occurs in cunjonction with situations perceived as critical for the maintenance of the elites' hegemony in that society (irrespective of "crimes rates"). He revisits the rich tradition inspired by Rusche's original hypothesis, but avoids the economist and instrumentalist temptations of that work. Evidence for the linkage between social structure and punishment is to be found in what he calls "vocabularies of punitive motive". He also shows that this theoretical reconstruction dovetails well with the crucial work of Foucault and other contributions to the sociology of law.

\section{REMERCIEMENTS}

Une version très éloignée de cet article a été présentée à l'atelier sur «le contrôle de la vie sociale» organisé à l'Institut Universitaire Européen de Florence (Italie) du 30 mai au 2 juin 1989. Ensuite, il a connu de trop nombreuses versions pour que je puisse toutes les mentionner. Je voudrais cependant remercier Edwin Lemert, Alvaro Pires, Ruth Rosen, Roger Rouse, Georges van den Abbeele, John Walton et les lecteurs anonymes pour leur précieux commentaires. Je voudrais également remercier, pour leur hospitalité et leur soutien, l'Institut Universitaire Européen qui m'a accueilli en 1988 et 1989, et le Davis Humanities Institute qui m'a reçu en 1990 et 1991.

À l'occasion de l'inauguration de l'«année judiciaire» 1975 à Turin, en Italie, le Procuratore Generale, Reviglio delia Veneria, formulait dans son discours pour l'ouverture le vœu suivant:

Que la tension sociale diminue ainsi que les grèves (industrielles) provoquées par les très graves conflits de travail; qu'un terme soit mis à l'agitation anarchiste et aux actes d'insubordination civile; que nous parvenions à ralentir l'effarante montée de la criminalité; que la paix et l'harmonie finissent par reprendre le dessus dans le cour des Italiens, afin qu'à l'unisson nous entreprenions de sortir de l'abîme dans lequel

1. Professeur, Department of sociology, University of California, Davis, California 95616, USA. 
nous nous enfoncions pour nous tourner à nouveau vers le progrès et la justice. (traduction libre; cité dans Insolera, 1975, p. 308-309).

Ce discours illustre bien le type de déclarations qui font autorité et que des personnes influentes émettent habituellement afin de donner au public le sentiment qu'elles poursuivent un but et une orientation définis. Aux États-Unis, la verve oratoire avec laquelle le président Spiro Agnew décrivait les conflits sociaux des années soixante fournit un autre exemple de ce type de réthorique:

Lorsque je parle des fauteurs de trouble, je pense aux agresseurs et aux criminels de la rue, aux assassins de dirigeants politiques, aux déserteurs et à ceux qui brûlent le drapeau, aux militants des campus, aux agitateurs et aux manifestants qui agissent contre les candidats aux fonctions officielles, ainsi qu'aux pillards et incendiaires des villes. (Traduction libre, cité dans Braithwaite, 1980, p. 198).

Comme le remarque Braithwaite, cette extravagante association de termes qui désignent une forme de déviance comporte un dessein idéologique (Braithwaite, ibidem). J'aimerais exposer ici un point de vue qui servira à formuler des hypothèses susceptibles d'expliquer les résultats actuels des découvertes et d'orienter la recherche à venir. Il s'agit de l'idée selon laquelle une plus grande sévérité du châtiment est en partie tributaire de la gestion idéologique des périodes de crise par les élites au pouvoir (crises qui peuvent survenir pour maintes raisons mais qui sont ainsi qualifiées par ces élites), quels que soient les taux fluctuants des comportements criminels officiellement enregistrés. Cette plus grande sévérité dans le châtiment est la résultante d'une relation particulière entre les structures sociales et une activité humaine précise, à savoir l'activité de punir. Cette relation est établie par ce que j'ai appelé les «vocabulaires de la motivation punitive" ("vocabularies of (punitive) motive"), vocabulaires dont la nature et le sens varient en fonction des modifications du contexte historique. (Mills, 1940; Melossi, 1985; Melossi 1990, p. 140-154).

Ce point de vue peut aussi expliquer le fait que, dans des systèmes juridiques différents et au sein de réalités nationales distinctes, les variations des taux d'incarcération au fil des années semblent être statistiquement liées aux modifications du contexte économique, sans pour autant qu'elles soient reliées à des variations des taux de criminalite, comme on pourrait s'y attendre. D'après moi, la notion théorique centrale à laquelle il faut faire appel pour comprendre ces associations statistiques est celle d'hégémonie. En me référant aux célèbres déclarations de Gramsci 
(1929-1935), j'entends par là la suprématie culturelle d'une vision du monde adoptée par les membres d'une société donnée afin d'organiser les rapports sociaux en vigueur à un moment (par exemple l' «éthique protestante » pendant la période de transition entre la destructuration de la société médiévale et la montée du capitalisme, comme le relate si bien Weber (1904-1905)). Les vocabulaires à motivation punitive ne sont rien d'autre que des articulations des motifs qui s'inscrivent dans ce type de vision globale du monde (Weltanschauungen) concernant l'activité sociale qui consiste à infliger des châtiments légaux.

Plus précisément, j'avance que ceux qui font partie de l'élite au pouvoir et qui sont "autorisés » à identifier et à étiqueter les problèmes sociaux (Gusfield, 1981 ; Spector et Kitsuse, 1977; Becker, 1963) et que je nomme «élites morales» ont une propension à réagir à des situations qu'ils perçoivent comme menaçantes pour les structures politiques, socio-économiques et culturelles qu'ils associent à la défense et à la promotion de leur propre hégémonie. Ce faisant, je m'appuie aussi sur la tradition sociologique selon laquelle le châtiment est le véhicule idéal pour transmettre des messages sur la moralité de la société (Durkheim, 1895; Mead, 1917-1918; Erickson, 1966).

Le but de cet article est donc de développer cette perspective théorique sur laquelle j'aimerais étayer l'hypothèse suivante: dans une société donnée, le processus d'incarcération accrue coïncide avec des situations perçues comme cruciales pour le maintien de l'hégémonie des élites (indépendamment des «taux de criminalité»). Dans le cadre de cette hypothèse, je voudrais souligner que, ce qui importe, ce n'est pas la situation de crise prétendument «objective", soit une situation jugée telle par un observateur neutre (un sociologue, par exemple), en fonction d'indicateurs sans rapport avec les critères d'évaluation des élites. Ce qui importe plutôt, c'est le fait que les élites perçoivent une crise comme existante ou imminente, l'interprétation morale qu'elles présentent de la situation et la réaction qui s'impose selon elles.

Je pense qu'à travers la formulation de cette hypothèse nous devrions être en mesure d'expliquer les résultats de la recherche mentionnée ci-dessus concernant la relation entre le crime, le châtiment et l'économie. Pour ce faire, cependant, il faut considérer les indicateurs de changements économiques dont se sert cette recherche comme des indicateurs de la perception des élites d'une situation de crise sociale, considération fondée sur le postulat selon lequel les périodes de difficultés économiques et de crises hégémoniques sont habituellement (mais pas nécessairement) liées. Évidemment, il serait recommandé 
d'utiliser des indicateurs directs de la gestion idéologique des élites pour ces périodes afin de mettre à l'épreuve l'hypothèse décrite plus haut. Dans la dernière partie de cet article, je propose un ensemble d'indicateurs «directs » de ce genre pour les vocabulaires à motivation punitive des élites.

Je soutiens l'idée qu'en procédant de la sorte nous serions aussi à même de redécouvrir la riche tradition d'analyse inspirée de l'hypothèse originale de Georg Rusche selon laquelle les modalités et conditions du châtiment étaient perçues comme étant tributaires de la structure sociale et spécialement du marché de l'emploi (Rusche, 1933; Rusche et Kircheimer, 1939). Il devrait être possible d'y parvenir tout en évitant les tentations économistes et instrumentalistes qui ont marqué l'œuvre de Rusche et de Kircheimer (Melossi, 1978, 1980) et certaines des études qui ont développé leurs analyses au cours des 10 à 15 dernières années (voir partie suivante).

Dans cet article, $\mathrm{j}$ 'analyse tout d'abord brièvement les études portant sur les rapports entre le châtiment et l'économie. Je montre ensuite comment les principaux résultats peuvent s'expliquer par une théorie mettant l'accent sur le rôle de l'hégémonie et son articulation à travers les vocabulaires à motivation punitive. Je vais jusqu'à démontrer que cette reconstruction thérique s'accorde parfaitement avec d'importants travaux de sociologie du droit et l'auvre décisive de Michel Foucault sur le châtiment (1975). Enfin, je décris un indicateur direct pour les vocabulaires à motivation punitive des élites dans le cadre sociohistorique concret de l'exemple du système pénal italien de la période qui va de la post-unification à l'heure actuelle.

\section{LA RECHERCHE SUR LE CHÂTIMENT ET LA STRUCTURE SOCIALE}

Après la résurrection classique de Rusche et Kircheimer en 1969 , de nombreux chercheurs ont mis les hypothèses de Rusche à l'épreuve en utilisant des données quantitatives longitudinales sur l'emprisonnement, le système de justice criminelle, la criminalité et l'économie (généralement le chômage). Les lieux, les époques et les indicateurs utilisés dans ces études variaient considérablement. Dans l'une des premières, Ivan Jancovic a employé les statistiques aussi bien fédérales que celles des différents états sur la prison aux États-Unis pour la période de 1926 à 1972 et est parvenu à la conclusion suivante : «La relation entre le chômage et l'emprisonnement était positive et 
statistiquement significative, indépendamment du volume de l'activité criminelle» (1977, p. 101). Il a toutefois relevé deux exceptions, à savoir les années correspondant à la grande dépression et les données sur les prisons du gouvernement fédéral antérieures à 1960. Mathew Yaeger est parvenu à des conclusions similaires dans son étude sur le milieu américain de 1972 à 1974 (1979), et Harvey Brenner déclarait: «Un pour cent d'augmentation du chômage qui se prolonge durant une période de six ans, aux États-Unis entre 1935 et 1973, coïncide avec un accroissement approximatif de 3,340 entrées en prison » (1976, p. 5-6). James Inverarity et Daniel McCarthy ont établi, compte tenu des variations de la criminalité, que les taux d'incarcération au niveau des différents États américains entre 1948 et 1981 étaient directement liés au taux de chômage et augmentaient en fonction de celui-ci (1988). David Greenberg a noté un lien étroit entre les niveaux d'incarcération et le taux de chômage au Canada entre 1949 et 1959 (1977). Il n'a cependant pas été en mesure de constater le même lien dans le cas de la Pologne « socialiste » d'après guerre. Pour expliquer cette disparité, il propose que, dans le cadre de la Pologne, les changements des taux d'incarcération ont pu être motivés par des considérations politiques et réalisés par le recours régulier à des amnisties accordées par le pouvoir exécutif (1980). (Cette suggestion rappelle l'étude de Richard Berk et ses collaborateurs qui sont parvenus à la conclusion que la mise en liberté conditionnelle (parole) était utilisée sciemment en Californie entre 1907 et 1977 pour contrôler la population de détenus, système qui a finalement été mis en échec lorsque le corps législatif a rendu la liberté conditionnelle impossible dans un nombre considérable de cas (Berk et al., 1983)).

De plus, l'Europe occidentale fournit matière à étayer l'hypothèse selon laquelle il existerait une corrélation entre l'économie et le niveau d'incarcération. Après avoir examiné des données ponctuelles pour l'Angleterre et le Pays de Galles pour la période allant de 1957 à 1981, Steve Box et Chris Hale ont conclu que «le nombre total d'individus faisant l'objet d'une sentence immédiate d'emprisonnement était relié de manière significative au niveau de sous emploi, même en tenant compte des niveaux de criminalité et du pourcentage des condamnations» (1944, p. 218). En Suisse, entre 1890 et 1941, Martin Killias et Christian Grandjean ont détecté un lien entre le chômage et le nombre de condamnations et entre le chômage et le nombre de personnes en attente de jugement (1986), mais ils n'ont pas été capables de contrôler la variable relative au taux de criminalité. Dans mon analyse de données italiennes couvrant la période de 1896 à 1965, il m'a été possible de 
trouver un rapport direct entre les fluctuations du cycle des affaires et les taux d'emprisonnement mais pas des condamnations, en tenant compte du taux de criminalité (Melossi, 1985). (En d'autres termes, dans un système comme celui de l'Italie où à peu près $50 \%$ des détenus attendent généralement leur jugement, les changements économiques semblent avoir un rapport avec le nombre des détenus qui attendent un jugement mais pas avec le nombre de ceux qui purgent une peine). Bernard Laffargue et Thierry Godefroy qui ont examiné les données françaises entre 1920 et 1985 ont découvert que le chômage était lié au taux đ'incarcération mais pas au taux de criminalité (1989). Exception notoire, le travail de Willem de Haan qui tenta de reproduire les études de Box et Hale en utilisant des données hollandaises (1990, p. 36-63). Bien qu'il se soit cantonné à examiner une courte période de temps (1975-1987), De Haan a tiré la conclusion qu'en Hollande, pour la période en question, il n'existait aucun rapport entre l'accroissement du chômage et celui du taux d'emprisonnement (alors que, par ailleurs, l'accroissement du taux de chômage et celui du taux de criminalité étaient statistiquement liés).

Pour parler d'un cas que ni soit ni américain ni europén, à savoir celui de l'Australie, notons que John Braithwaite, qui a analysé les tendances historiques des taux d'incarcération sur le long terme pour les Etats de New South Wales et de Victoria, a découvert un certain rapport entre une tendance historique au long terme de décarcération marquée et une diminution graduelle du chômage (1980). Enfin, dans un récent essai, Theodore J. Chiricos et Miriam A. Delone examinaient les résultats de quelque 47 études sur le rapport entre les indicateurs du marché de l'emploi et l'emprisonnement. Ils ont décelé une très grande prédominance d'études qui démontrent un lien statistique étroit, dans le temps, entre les fluctuations du contexte économique et l'incarcération, lien qui se confirmait compte tenu des taux de criminalité (1992).

Ainsi, dans quantité de systèmes juridiques distincts, il s'avère que les écarts entre les taux d'incarcération sont liés aux variations des indicateurs économiques sans (ou presque sans) que les taux globaux (enregistrés officiellement) de comportements criminels n'y jouent un rôle prépondérant. Il convient de noter, bien entendu, que ces analyses employaient des types de données disparates, des modèles très différents et des techniques statistiques tout aussi dissemblables, et ne peuvent donc pas être considérées comme des vérifications similaires de mêmes hypothèses sur différents échantillons. En outre, le lien statistique entre les modifications du contexte économique et le taux d'incarcération correspond généralement à une petite fraction de la variance 
dans ces derniers. En d'autres termes, ce lien ne peut en aucun cas consolider une explication linéaire qui relierait les changements dans les chiffres de l'emprisonnement exclusivement aux modifications du contexte économique. Il conviendrait de s'intéresser à d'autres variables comme celles de nature bureaucratique et démographique proposées par Richard Berk et al. (1983), et Al. Blumstein et al. (1980). Il me semble, cependant, que des résultats identiques obtenus en dépit de structures sociales et de systèmes pénaux très différents ne peuvent guère être écartés sous prétexte qu'il s'agirait simplement d'aberrations statistiques. Je pense qu'ils indiquent une tendance des systèmes de justice criminelle à traiter les cas sans perdre de vue certains types de changements sociaux que reflètent généralement les modifications des indicateurs économiques. En d'autres termes, une fois qu'il a fallu écarter l'explication hâtive selon laquelle un accroissement de la fréquence du châtiment résulterait d'une augmentation de la criminalité (Greenberg, 1977) dans la mesure où le rapport entre changement économique et incarcération ne semblait pas lié au taux de criminalité, la question qui se pose est la suivante: comment ce type de lien s'établitil ? C'est là la question théorique centrale de cet article à laquelle la recherche n'a jusqu'à présent pas fourni de réponse satisfaisante.

\section{2. ÉLITES MORALES, PRATIQUES DISCURSIVES ET STRUCTURE SOCIALE}

Dans le climat politique et culturel des années soixante-dix, les résultats des recherches que nous évoquions suscitaient des hypothèses qui tenaient d'une vision plutôt conspiratrice des rapports sociaux. Jankovic, par exemple, énonçait le postulat selon lequel le système pénal constituait une manière de contrôler l' «excédent de travail » produit en permanence par la machine capitaliste (Jankovic, 1977, p. 95). Ce type d'hypothèses se fondait sur l'idée que les établissements pénitentiaires allaient de pair avec l'exploitation capitaliste par l'intermédiaire d'un concept passe-partout: l'«État». Dans son célèbre essai sur les «appareils idéologiques d'État», Louis Althusser (et ceux qui lui ont embôté le pas) utilisait le concept d'État comme un outil pratique qui permettait de mettre en valeur l'homologie entre la structure de l'usine et celle de la prison, ou de l'école (1970). Le problème est que l'«État» en question est seulement une idée abstraite qui exprime l' " orientation étatique " de politiciens, enseignants, directeurs de prison et tous les individus qui ont un rapport avec l'État (Melossi, 1990). De quelle manière l'enseignant, le directeur de prison, le journaliste, le 
politicien ou le capitaliste entendent-ils cette homologie et comment contribuent-ils à son édification à travers les canaux institutionnels et culturels qui se trouvent à la fois à l'intérieur et à l'extérieur de l'«État»?

Cette question pose les jalons du problème de la reproduction idéologique, processus qui se situe entre «la tradition de toutes les générations passées [qui] pèse comme un cauchemar sur le cerveau des vivants" (traduction libre; Marx, 1852, p. 595) et l'esprit d'entreprise innovateur des élites intellectuelles qui ont une influence sur la production et la reproduction de constructions symboliques, mais pas dans des «circonstances qu'elles choisissent elles-mêmes» (traduction libre; Marx, ibidem). Les vocabulaires employés par les élites ne sont donc pas une invention soudaine et fortuite. Il s'agit plutôt de la décantation figée d'élaborations intellectuelles de longue date auxquelles on prête de nouvelles formes pour accompagner, ou plus précisément organiser, l'émergence d' "ensembles d'actions situées» ou de «mondes vivants» envisagés par ces élites.

Je m'engage ici dans une voie qui nous mène au-delà des perspectives dualistes de la réalité sociale. Mon propos est en effet de mettre en relief le fondement constitutif des discours et pratiques, le rôle crucial de la communication sans lequel aucune organisation n'est possible. C'est là une voie que Marx a déjà indiquée dans le passage de l'Idéologie allemande où le langage est défini comme une "conscience pratique» qui «naît seulement du besoin... de rapports avec d'autres hommes» (traduction libre; 1845, p. 158), déclaration que je trouve difficile à concilier avec la philosophie dualiste rigide qui sous-tend les concepts de matérialisme historique et d'idéologie abordés dans le même texte. Le fait est que nous utilisons la communication pour donner une forme à l'organisation sociale qui correspond à la manière dont les membres de ces organisations les décrivent. Nous «faisons des choses avec les mots» d'une manière bien plus radicale que ne le laissait entendre J. L. Austin (1975) et la théorie des actes de langage (speech act) en général. Les penseurs pragmatistes, notamment Georges Herbert Mead, ont présenté ces concepts de la façon la plus fondamentale. Pour Mead, la constitution du sens au niveau le plus fondamental, et donc de la pensée, provient de l'interaction communicative dont elle est indissociable. C'est la forme la plus fondamentale de coopération humaine $(1934$, p. 68-82). Voilà pourquoi, au niveau le plus large, «le processus de communication est plus universel que la religion universelle ou le processus économique universel, dans le sens où il fonde ces deux derniers» (Mead, 1934, p. 259). Plus tard, C. Wright Mills, 
s'appuyant sur les résultats auxquels était parvenu le courant pragmatique, établit une relation entre ce qu'il appelait «actions situées» et les «vocabulaires de motifs» $(1930,1939)$.

Certes, dans un monde dominé par la division du travail, il peut se créer un hiatus entre les discours façonnés par les élites intellectuelles et la façon dont les élites au pouvoir se comprennent elles-mêmes. Toutefois, à commencer par le texte classique de C. Wright Mills, les écrits sur les élites au pouvoir ont expliqué la manière dont ce hiatus pourrait être évité en centralisant les médias, en encourageant la socialisation dans les écoles des élites, etc. (Mills, 1956, p. 106-107, 298-324; Bourdieu, 1989). C'est l'aspiration des élites intellectuelles que de donner une cohérence à l'organisation sociale dominée par l'élite au pouvoir dont elles font partie intégrante. La vocation propre des élites morales est de rendre cohérentes les positions morales de la société et de s' «approprier» ainsi, pour utiliser l'expression de Gusfield (1981), certains domaines particuliers de la vie sociale. ${ }^{2}$

Je n'entends certes pas par ces propos tenter de nier que les élites émergent à partir de conflits, notamment dans des situations «critiques». En fait, il faudrait voir les discours conflictuels comme des propositions conflictuelles visant à organiser la société ou des secteurs spécifiques de la société, propositions émanant d'élites, ou de groupes aspirant à le devenir, qui se trouvent en conflit. L'apparition de projets idéologiques rivaux correspond aux divisions au sein des différents groupes d'élites disposant de đifférents types d'audiences. Les recherches sur le contrôle social ont montré la relation existant entre certaines formes de communication et les «autres», les "publics» ou les «groupes de reférence» auxquels ces formes s'adressent (Shibutani, 1962; Melossi, 1990, p. 140-145). En même temps, ce que je viens de

2. Le concept d'élite morale que je propose ici est plus vaste que le concept d' «entrepreneur moral » de Howard Becker dans le sens ou je m' intéresse plus particulièrement à la relation entre les élites et les traditions morales générales qui forment les bases des «campagnes » déterminées. Le discours moral ne peut se concevoir simplement comme étant promu et établi par des organes ou individus particuliers (comme le Federal Bureau of Narcotics dans le cas tant débattu de la loi de l'impôt sur la marijuana (Marijuana Tax Act) de 1937; voir Becker, 1963 et Galtiher et Walker, 1977). Il doit plutôt être conceptualisé comme discours continu auquel des individus et organes peuvent sans doute contribuer mais qu'ils ne peuvent pas créer de toutes pièces, même lorsqu'il coïncide avec les perturbations historiques les plus profondes. Citons a ce propos le mot heureux d'Arthur Bentley : «Les descriptions, observations et considerations se présentent à moi comme des actions, non d'individus singuliers, mais comme les signes mouvants des comportements verbaux des hommes, avançant et reculant avec les siècles. $*(1953$, p. 12) 
souligner ne devrait laisser aucun doute sur le fait que le conflit entre des «projets » rivaux d'organisations sociales ne se livre pas au seul niveau dominant ainsi que le laissaient entendre les hypothèses dualistes qui sous-tendent l'idée de la relation de l'infrastructure et de la superstructure, production d'idéologie ou fabrique d'institutions «ancillaires» comme je les ai appelées dans des ouvrages précédents (Melossi, 1976). Ce conflit se situe plutôt à l'intérieur aussi bien de l'organisation sociale que de la communication sociale, simultanément et concomitamment.

\section{ILLÉGALISMES, CRIMES NORMAUX ET CONTRÔLE SOCIAL}

Les actions des élites morales définissant des situations comme critiques et appelant des remèdes particuliers devraient être donc considérées comme des "projets " lancés à la société, projets dont le sort dépend de l'issue de la bataille qui gronde, pour emprunter l'expression de Michel Foucault dans sa conclusion de Surveiller et punir (1975, p. 315) et que j'oserais appeler bataille pour l'hégémonie. Il est important de noter cependant que cette bataille n'est pas le sujet central du livre de Foucault qui se consacre plutôt à l'analyse de «discours qui sont eux-mêmes des éléments de [...] stratégie» (Foucault, ibidem). De fait, le discours sur la discipline qui domine l'œuvre décisive de Foucault pourrait être bien classé dans la catégorie des projets d'hégémonie, même si Foucault semble parfois s'y référer comme à une hégémonie dejà réalisée. L'analyse des principales sources de Surveiller et punir révélerait en fait que le sujet principal est le discours sur la pratique et non le discours en pratique. Nous nous trouvons en fait devant des Utopies de contrôle, des projets directeurs des réformateurs pénaux, ou des «recettes du livre " de diverses institutions sociales ${ }^{3}$.

3. Le contraste entre une description de la discipline pénale limitée à ses règles d'application (qui est au coeur de la reconstruction de Foucault) et la vie réelle à l'intérieur de l'établissement est rendu à merveille à travers deux récits tout à fait différents sur la discipline à la colonie de Mettray. Dans Surveiller et punir, Foucault fait coïncider l'avènement d'un nouveau style perfectionné de contrôle disciplinaire avec l'ouverture officielle, le 22 janvier 1840 , de la colonie de Mettray pour jeunes délinquants, \& la forme disciplinaire à l'état le plus intense, le modèle où se concentrent toutes les technologies coercitives du comportement» (1975, p. 300). Comparez la déclaration de Foucault avec les réflexions de l'un des criminels les plus éloquents de notre siècle, Jean Genet, qui a fait le commentaire suivant à propos de son séjour dans cette maison de correction plusieurs annees plus tard:

C'est plus tard que je découvris les vertus de la discipline, par les règles formelles, 
Lorsque l'on évoque l'histoire du contrôle social en tenant uniquement compte des projets directeurs réformateurs du système pénal, on éclipse la question du contrôle social dans la pratique, à savoir la question de l'hégémonie, qui est le résultat des processus sociaux dans lesquels les rôles contradictoires joués par différents groupes, classes, agences et organisations sont essentiels. D'un point de vue historique, le projet d'un système disciplinaire caressé par les élites était condamné à se heurter à la résistance de ceux qui refusaient de se laisser modeler au gré des nouvelles priorités, se raccrochant aux anciens modes de vie, et pour lesquels les nouveaux vocabulaires bourgeois, qui comprenaient des mots comme droit (de propriéte), travail, crime, police et contrôle administratif, apparaissaient comme tout à fait étrangers, si ce n'est franchement hostiles (Hobsbawm, 1959; Hay, 1975; Linebaugh, 1976; Flacks, 1988).

Il me semble que cela explique en partie pourquoi, après avoir consacré quelque 268 pages de Surveiller et punir (1975) à la reconstitution des origines du système disciplinaire fondée sur les projets directeurs des réformateurs, Michel Foucault remarque que, depuis le début, le projet de prison «a été dénoncé... comme le grand échec de la justice pénale» (1975, p. 269). Non seulement les prisons n'ont pas réussi à réformer les criminels, mais elles les ont rendus plus durs, plus malins et plus désespérés, devenant de véritables « universités du crime !». À la fin de Surveiller et punir, Foucault choisit donc de passer d'un discours principalement axé sur la vision de l'emprisonnement comme formation à la discipline, à une vision selon laquelle la fonction sociale de l'incar-

appliqué à la colonie de Mettray. Pour devenir colon (c'est ainsi que l'on appelait les enfants), j'ai do me forcer. Comme la plupart des petits voyous, j'ai fait, sans m'y arrêter, beaucoup des actions qui faisaient de nous des colons [...] Afin de me tirer du désespoir qui me prenait lorsque je me perdais dans mes pensées, je me suis, à mon insu, élaboré une ferme discipline. Le mécanisme était à peu près le suivant (j'y ai eu recours depuis): à chaque accusation portée contre moi, même si elle était injuste, je répondais oui du fond de mon cœur. À peine avais-je prononcé ce mot, ou une phrase qui avait ce sens, que je sentais le besoin irrépressible de devenir ce que l'on m'avait accusé d'être. J'avais seize ans. Le lecteur l'aura compris: je n' ai laissé dans mon cơur aucune place où le sentiment de mon innocence puisse se réfugier. J'avouais être le lâche, le traŝtre, le voleur, le vaurien qu'ils voyaient en moi. Il est possible d'accuser sans preuve mais il me semble que pour avoir été reconnu coupable, il faut que j'aie perpétré les actes qui font les traîtres, les voleurs ou les lâches; or cela n'était pas du tout le cas: en mon fort intérieur, avec un peu de patience, j'ai découvert, par la réflexion, des raisons valables de mériter ces noms. Et cela me bouleversa de savoir que j'étais composé d'impuretés. Je suis devenu vil. Peu à peu, je me suis habitué à cet état. Je le reconnais ouvertement. Le mépris que l'on me portait se changea en haine: $j$ 'avais réussi. (Traduction libre; Genet, 1949, p. 175-176). 
cération a été, depuis le départ, de transformer les «illégalismes» en « délinquance»:

[...] Il faudrait alors supposer que la prison et d'une façon générale sans doute, les châtiments ne sont pas destinés à supprimer les infractions; mais plutôt à les distinguer, à les distribuer, à les utiliser; qu'ils visent, non pas tellement à rendre dociles ceux qui sont prêts à transgresser les lois, mais qu'ils tendent à aménager la transgression des lois dans une tactique générale des assujettissements. La pénalité serait alors une manière de gérer les illégalismes, de dessiner des limites de tolérance, de donner du champ à certains, de faire pression sur d'autres, d'en exclure une partie, d'en rendre utile une autre, de neutraliser ceux-ci, de tirer profit de ceux-là. Bref, la pénalité ne «Iéprime» pas purement et simplement les illégalismes; elle «différencierait », elle en assurerait l'«économie" génerale. Et si on peut parler d'une justice ce n'est pas seulement parce que la loi elle-même ou la manière de l'appliquer servent les intérêts d'une classe, c'est que toute la gestion différentielle des illegalismes par l'intermédiaire de la pénalité fait partie de ces mécanismes de domination. Les châtiments légaux sont à remplacer dans une stratégie globale des illégalismes. L' «échec» de la prison peut sans doute se comprendre à partir de là. (Foucault, 1975, p. 277)

Les observations de Foucault sur l'echec de la prison et sur ses buts intentionnels, si éloignés de ses fonctions «réelles», peuvent difficilement être réduits à un nouveau but instrumental et intentionnel: le contrôle de la classe ouvrière (Garland, 1990, p. 6). Cela rappelle plutôt le fameux chapitre de Durkheim dans Les règles de la méthode sociologique consacré à la distinction entre le normal et le pathologique en vertu de laquelle une société exempte de crimes serait inconcevable car ce serait une société sans normes (1895). Le projet central de la pénalité serait alors son usage symbolique comme une pièce de théâtre moralisante ou plutôt comme une gazette officielle de la moralité. Foucault partage avec d'autres penseurs sociaux, essentiellement Marx et Weber, l'idée qu'en toute logique le contrôle de la classe ouvrière ne peut guère être séparé de l'univers moral et idéologique dont elle se nourrit et auquel les élites au pouvoir font appel pour légitimer leur gouvernement. C'est pourquoi les «stratégies» dont parle Foucault dans Surveiller et punir ont souvent l'air de n'appartenir à personne. Ce sont des stratégies "systémiques», des façons dont la fonctionnalité systémique d'une institution donnée, comme la prison, est assurée socialement. 
En d'autres termes, ces émotions, ces «élans de sentiments communs " comme les appelle Garland dans son résumé de la position de Durkheim sur le châtiment $(1990$, p. $8-9)$, sont produits par la société tant pour ce qui est de leur importance que de leur orientation. Ils expriment leur indignation face à certains comportements et non face à d'autres. Comme nous l'avons appris à travers la sociologie de la déviance et du contrôle social (Becker, 1962 ; Gusfield, 1981 ; Erickson, 1966 ; Cohen, 1972), il apparaît que, d'une part, le caractère symbolique de la réaction sociale peut se distinguer d'une réaction intentionnelle et instrumentale, alors que, d'autre part, il dépend de sensibilités politiques et de classe précises, socialement hégémoniques dans des circonstances sociales et historiques données.

Ce que Foucault appelle transformer les illégalismes en délinquance semblerait alors être la façon dont fonctionne ordinairement un système de justice pénale dont la majorité des membres partagent les idéologies, les préjugés et les préférences de la société dans son ensemble et dont le but n'est pas tant d'éliminer la criminalité (comme le reconnaîtrait n'importe quel représentant de la justice criminelle) mais de la «contenir» dans des limites qui soient «acceptables» «normales» pour une sociêté donnée, à un moment précis. En d'autres termes, la police et les représentants de la justice criminelle font, bon gré mal gré, partie intégrante d'une structure de pouvoir et d'idéologie. Je veux dire par là que leur façon de comprendre la lutte contre la criminalité est étroitement liée à leur façon d'appréhender la société dont ils sont issus, et donc leur relation avec elle. Cela ne signifie pas qu'ils aient l'«intention " de contribuer à transformer les illégalismes en délinquance. Cela signifie tout simplement que, dans leurs activités quotidiennes, ils auront des choix pratiques à faire sur un éventail de comportements qui pourraient être socialement définis comme étant des actes criminels, et ils devront faite ces choix en fonction du point de vue de la société ainsi que des structures professionnelles et institutionnelles dont ils font partie. Leur travail consiste essentiellement à préserver l'équilibre précaire entre la production sociale d'activités habituellement identifiées comme faisant partie de ou liées à la «criminalité » (les stupéfiants, les organisations criminelles, la prostitution, les paris, la corruption parfois les crimes en col blanc, etc.) et la circonscription de ces activités dans des limites qui évitent que le «bateau ne coule». Bien qu'il leur faille tolérer la présence limitée de ces activités dans la sociêté de facto, ils ne doivent pas permettre qu'elles dépassent un point qui ferait penser que la société a modifié son seuil de tolérance «officiel ». 
C'est pourquoi il faut compléter les analyses de Durkheim et de Foucault en utilisant la notion de «normalité » avancée par David Sudnow (1965). D'après l'œuvre de Sudnow, les «crimes normaux» sont des crimes produits et gérés par la société sans trop de problèmes. Ces crimes ne sont toutefois pas normaux seulement au sens où l'entend Sudnow (c'est-à-dire qu'ils sont immédiatement reconnaissables comme étant des événements qui font partie d'une phénoménologie familière de comportement criminel et qui sont pris en charge par des membres compétents du système de justice pénale à travers un inventaire standardisé de pratiques linguistiques et procédurales routinières); ils sont aussi normaux au sens que ce sont des événements auxquels on est en droit de s'attendre dans la société dans laquelle on vit, selon l'interprétation que se font les élites morales de cette société.

Le «crime», tel que nous le connaissons, est donc le produit d'un certain nombre d'institutions sociales dont celles qui sont chargées de la police, de la justice criminelle, du châtiment et de la correction occupent le premier plan. Le processus de criminalisation (et de châtiment) peut certes produire un subtil effet de contrôle social mais pas nécessairement de contrôle social de la criminalité. Le spectacle du châtiment a un effet de contrôle social sur ceux dont il est improbable qu'ils courent le risque de se voir classés dans la catégorie «criminelle». Parmi les spectacles visant à engendrer l'hégémonie, l'incarcération montre à un plus large public à la fois le comportement social acceptable dans un contexte historique donné et l'infamie de ceux qui ne s'y conforment pas.

Cette «tactique d'assujettissement», pour utiliser la formule de Foucault, ne marche cependant pas forcément uniquement dans le sens de la sévérité. Du fait précisément que les préoccupations et les sensibilités des élites politiques sont associées au contrôle du gouvernement même si c'est au sens général du «il tenere lo stato» dont parlait avec beaucoup de finesse Machiavel - ces préoccupations peuvent être mieux exprimées, dans certaines circonstances historiques et culturelles, à travers la clémence, la compréhension et la «tolérance». Ainsi, la série de meurtres, apparemment sans rapport, de candidats à des fonctions officielles qui se sont produits pendant les élections locales de mai 1990 dans les provinces napolitaines et calabraises de l'Italie du Sud, qui étaient, à n'en pas douter, l'œuvre du crime organisé, a été décrite par l'ex-premier ministre italien, le chrétien démocrate Giulio Andreotti (dont le parti est sorti vainqueur de ces élections) comme "quelques cas de violences certainement sérieux» (traduction libre) 
dont le sens ne devrait cependant pas être «exaspéré ni amplifié » ${ }^{4}$ (traduction libre). On ne peut s'empêcher de se rappeler un leitmotiv du méridionalismo italien sur les relations nord-sud en Italie. Selon les auteurs méridionalisti, depuis l'époque de l'unification italienne en 1861, les gouvernements conservateurs centraux de Rome ont adopté une attitude pour le moins ambiguë face à la présence d'organisations criminelles dans l'Italie du Sud, cette présence ayant traditionnellement contribué à la préservation d'un équilibre du pouvoir national capable de compenser la composition sociale et politique plus progressiste de l'Italie du Nord et du Centre, exemple apparemment parfait pour étayer l'argumentation de Foucault (Gramsci, 1926; Salvemini, 1963).

\section{LES ÉLITES MORALES ET LES CHANGEMENTS DE VOCABULAIRE À MOTIVATION PUNITIVE: LE CAS DES PROCURATORI GENERALI ITALIENS}

Le processus social mis en branle par les élaborations culturelles des élites morales face à une situation de crise sociale - qu'il s'agisse d'un ralentissement économique, d'une guerre, d'un changement de régime politique ou même d'une "vague de crimes» - est apparenté au processus de redéfinition de normes qui s'accompagne d'une redefinition d'identité ou des facettes de l'identité du groupe concerné, decrit par Kai Erickson dans Wayward Puritans (1966). De la même manière qu'une «vague de crimes» donnée est utilisée à l'intérieur du groupe afin de discuter et de redéfinir publiquement des aspects déterminés du cadre normatif d'une société donnée, on utilise la préoccupation relative à la criminalité en général pour redéfinir le ton moral général d'une société. C'est une démarche continue à travers laquelle la détermination

4. Passage extrait de la déclaration du premier ministre Giulio Andreotti le 25 mai 1990 devant la Chambre des députés italienne, telle qu'elle a été rapportée par le journal ll popolo, organe du Parti démocratique chrétien, le 26 mai 1990, à la page 32. On aurait pu s'attendre cependant à un changement dans la prise de position de $\mathbf{M}$. Andreotti depuis. En effet, ces épisodes n'ont été qu'une simple étape dans la montée de la violence, relicé à la compétition non seulement pour la suprématie sur le marché de biens illicites, mais surtout pour l'obtention de privileges publics. Cette monté de la violence a fait grimper les taux d'homicide dans ces provinces a un niveau très près de la moyenne américaine, ce qui est un événement rare pour les régions de l'Europe * civilisée *. Plus encore: même après le récent assassinat a Palermo, au cours de la campagne électorale, de son ami proche et un des politiciens les plus influents de Sicile, l'Honorable Salvo Lima, un membre du Parfement européen, la colère de M. Andreotti était encore dirigée vers ceux qu'il a nommé $i$ professionisti dell'anti-mafia (les * professionnels de l'anti-mafia * ; voir La Republica, le 14 mars 1992). 
du seuil du châtiment tend à être liée à l'attitude de la societé par rapport à la déviance en général.

Les projets visant à rendre les châtiments plus sévères devraient donc être considérés comme entrant dans le cadre des projets plus globaux de discipline sociale associés aux situations critiques, telles qu'elles sont définies par les paramètres de la pensée hégémonique, définition dans laquelle intervient généralement (mais pas nécessairement) la situation de l'économie (par opposition à un climat de tolérance que l'on associe à une situation de "paix sociale», qui est une situation d'hégémonie absolue, quels que soient les taux de criminalité). En d'autres termes, la corrélation entre les variations du contexte économique et les changements apportés au système pénal n'est qu'un cas particulier de lien plus général entre les crises sociales et les modifications des politiques pénales, lorsque les indicateurs économiques peuvent être considérés comme des indicateurs d'une situation de crise. Les exceptions mentionnées au début de cet article où le chômage (ou un quelconque autre indicateur économique) ne semble pas avoir de rapport avec les mesures d'incarcération peuvent donc s'expliquer puisque, dans des situations historiques et sociales données, les modifications du contexte économique peuvent ne pas être perçues comme entraînant des problèmes d'instabilité et de légitimation, ainsi qu'il en a probablement été de la Hollande au cours de ces dernières années (De Haan, 1990, p. 36-63; Lamp, 1989) ou de la Pologne socialiste (Greenberg, 1980). Cela peut également être le cas des sociétés où les crises politiques et la «délégitimation» ont une incidence sur les niveaux d'emprisonnement sans qu'il y ait forcément de rapport avec les crises économiques, comme le suggèrent les résultats de la recherche de Lucia Benacquisto selon laquelle il existe une corrélation historique entre les «flambées " d'incarcération et les situations de crise politique dans un certain nombre de pays (1989).

La manière dont les élites perçoivent une situation de crise a un effet sur les «vocabulaires à motivation punitive » hegémoniques à travers l'ensemble de la société d'une façon qui n'est pas nécessairement propagée par les institutions officielles mais plutôt culturellement, du fait de la force et du prestige des formulations intellectuelles élaborées par les élites. Selon Durkheim, l'État est «... l'organe par excellence de la pensée sociale (rationalisée)» (traduction libre), social s'entendant ici au sens de collectif selon les termes de l'auteur (Durkheim, 18981900). Ainsi, dans le cas de l'Italie, on a toujours confié aux plus hauts magistrats du Ministère Public la tâche d'inaugurer les «années judiciaires» avec des discours comme celui du Procuratore Generale Revi- 
glio della Veneria, dont j' ai cité un passage dans le paragraphe d'introduction de cet article. Il s'agit de déclarations de politique qui font autorité en matière de justice criminelle et de politique pénale. Elles font autorité dans le sens où, d'une part, elles constituent un résumé du climat moral caractérisant la perception des affaires criminelles et pénales dans un contexte social donné, et, d'autre part, elles fournissent aux membres des systèmes pénal et de justice criminelle une orientation morale, politique et professionnelle. Ces déclarations sont faites dans une atmosphère de grande solennité et sont largement débattues, commentées et considérées comme importantes (même lorsqu'elles ne font pas l'unanimité) par les praticiens du domaine aussi bien que par les profanes. Depuis leur introduction, après l'unification de l'Italie en 1861 , ces discours publics ont brossé un portrait détaillé de la situation de la «question criminelle» en Italie. À travers eux, les magistrats indiquent leur attitude, année après année face à des questions comme celles de la sévérité du châtiment, des réformes pénales, des «vagues de crimes », etc.

Alors que l'on pourrait s'attendre à ce que de telles déclarations se rallient aux formules les plus sûres techniquement et politiquement, ce qui est propre aux propositions technico-légales, ce n'est habituellement pas le cas. Ce sont plutôt des appels à la solidarité et au soutien de la communauté pour des choix qui, même s'ils sont exprimés dans un jargon juridique, reposent nonobstant sur des bases politiques et morales et doivent être présentés comme tels. Le juriste, lorsqu'il doit se tourner vers la communauté pour obtenir son approbation et son soutien, doit parler la langue de celle-ci. Ainsi, dans leurs discours, les Procuratori Generali défendent habituellement leurs positions en termes politiques ou plus précisément moraux. Lorsqu'ils abordent les "problèmes de justice», qu'il s'agisse de justice civile ou pénale, ils identifient les questions fondamentales et font des commentaires sur les solutions proposées par les législateurs et le gouvernement, ainsi que l'administration et les diverses forces de police. Ils replacent ensuite le tableau dans le cadre plus large d'une évaluation éthique globale du développement économique et social du pays. Cette partie analytique est habituellement suivie de propositions par lesquelles on soumet des réformes juridiques propres à l'attention des législateurs, réformes qui sont présentées comme étant les seules capables de combattre «la profonde crise» que traverse le système de justice, ainsi que la société italienne dans son ensemble (Insolera, 1975, p. 289-290).

Ces discours ne constituent, bien entendu, qu'une seule sorte d'indicateurs «directs» de la gestion des périodes de crise par les élites. 
Ils ne représentent qu'un simple maillon de la chaîne discursive qui relie des formes de comportements aussi éloignées que celles qui consistent à exposer des valeurs morales, faire des affaires et infliger un châtiment. Il va sans dire que d'autres indicateurs pourraient servir à reconstruire cette chaîne discursive plus efficacement. Il suffit de mentionner les documents législatifs, les reportages des médias, et les articles dans les magasines et journaux spécialisés. Toutes ces déclarations discursives ont peu de rapport avec ce que l'on entend généralement par «directive» bureaucratique, mais elles devraient être considérées comme des contributions et des mises au point d'un discours hégémonique existant sur les questions de moralité particulière jugées assez graves pour être traitées comme des questions capitales du point de vue pénal. Les mêmes discours des Procuratori Generali, par exemple, si la personnalité du Procuratore Generale concerné a son importance, ont tous en commun de refléter l'attitude d'un homme responsable qui expose un état de choses et tente en même temps de se faire le porteparole d'un "milieu de responsabilité " constitué de membres du gouvernement, de politiciens appartenant aux partis au pouvoir, de hauts magistrats, de professeurs de droit, de représentants militaires et religieux de haut rang. En effet une fois le discours prononce, il sert à signifier le jugement moral des organes institutionnels représenté par ces responsables, la magistrature, l'État, la nation, le pays, etc. Le discours même est amplifié et commenté à travers toute la hiérarchie des tribunaux et des structures policières d'un côté, des médias et de l'opinion publique de l'autre.

S'il est par conséquent possible que l'accroissement des taux d'incarcération soit influencé par les effets fortuits d'une série de microdécisions fondées sur des considérations telles que le fait que le contrevenant est sans emploi (Box et Hale, 1984), il est aussi vrai que ces microdécisions (émanant non seulement des tribunaux, mais aussi d'officiers de police, de législateurs, d'entrepreneurs moraux, etc.) ne sont pas prises dans le vide. Elles doivent se justifier tant légalement que moralement à l'intérieur d'un discours hégémonique dominant envers lequel ceux qui administrent les systèmes de justice criminelle et pénale se sentent particulièrement responsables (qu'ils partagent ou pas la sagesse des agencements politiques contingents de ces systèmes). Le concept d'un «vocabulaire à motivation punitive " hégémonique qui se modifie pourrait bien nous permettre non seulement d'éviter l'hypothèse d'intentions conspiratrices généralement attribuées à ceux à qui l'on confie la tâche de prendre ces décisions, mais, surtout, nous aider à 
mieux spécifier et exprimer le caractère cohérent de toutes ces microdécisions.

Les signes de crises (que les taux de criminalité en fassent partie ou pas) doivent être détectés et perçus comme tels par la société, c'est-àdire par les élites morales. Selon le moment et le lieu, on peut craindre le «terrorisme» plus que le «crime organisé», la «drogue» plus que les "actes de vandalisme de spectateurs de match de football», ou une «vraie guerre" pour corriger la déviance des régimes du lointain TiersMonde, comme le notait David Matza à propos de l'avant-dernière grande guerre américaine (1969. p. 145), plus qu'une «guerre contre la drogue " afin de corriger la déviance de notre propre "sous-classe" nationale. Les sensibilités fluctuantes de la société tendent en fait à varier en fonction des responsabilités de ses élites. Les membres de la société apprennent à croire que les préoccupations et les ennemis des élites sont les leurs. Ceci est loin d'être le résultat de conspirations. C'est une contribution pertinente et une partie intégrante de ce que nous appelons l' «hégémonie».

\section{BIBLIOGRAPHIE}

ALTHUSSER, L. (1969), Lénine et la philosophie, Paris, Maspero.

AUSTIN, J. L., (1975), How To Do Things With Words, Cambridge, Mass., Harvard University Press.

BECKER, H. S. (1963), Outsiders. Études de sociologie de la déviance, Paris, Métailie, (trad. française, 1985).

BENACQUISTO, L. (1989), «Outburst of Repression and Political Tension: A Comparative Analysis of the Use of Incarceration », exposé présenté à The American Sociological Association Meetings, San Francisco.

BENTLEY, A. F. (1953), "Epilogue». p. 210-213, in R. W. Taylor (ed.), Life, Language, Law. Essays in Honor of Arthur F. Bentley, Yellow Springs, Ohio, The Antioch Press.

BERK, R. A., MESSINGER, S. L., RAUMA, D. et BERECOCHEA, J. E., (1983), «Prisons as Self-Regulating Systems: A Comparison of Historical Patterns in California for Male and Female Offenders», Law and Society Review, vol. 17, p. 547-586.

BLUMSTEIN, A., COHEN, J, et MILLER, H. (1980), «Demographically Desaggregated Projections of Prison Populations », Journal of Criminal Justice, vol. 8 , p. 1-26.

BoURdiEU, P. (1989), La Noblesse d'État, Paris, les Éditions de Minuit. 
BOX, S. et HALE, C. (1984), «Liberation - Emancipation, Economic Marginalisation, or Less Chivalry *, Criminology, vol. 22, p. 473-497.

BRAITHWAITE, J. (1980), «The Political Economy of Punishment», p. 192-208, in E. L. Wheelright et K. Buckley, (Éds.), Essays in the Political Economy of Australian Capitalism, vol. 4, Sydney, Australian and New Zealand Book.

BRENNER, M. H. (1976), «Estimating the Social Costs of National Economic Policy: Implications for Mental and Physical Health and Criminal Aggression ", Paper $n^{\circ}$ 5, Joint Economic Committee, Washington, D.C., U.S. Government Printing Office.

CHIRICOS, T. G. et DELONE, M. A. (1992), «Labor Surplus \& Punishment: A Review and Assesment of Theory and Endence», Paper presented at State-of-theDot Conference on Inequality, Crime and Social Control, the University of Georgia, Athens, April 10-12.

COHEN, S. (1972), Folk Devils and Moral Panic. The Creation of the Mods and Rockers, New York, St. Martin's Press, 1980.

DE HAAN, W. (1990), The Politics of Redress. Crime Punishment and Penal Abolition, Londres, Unwin Hyman.

DURKHEIM, E. (1898-1900), Professional Ethics and Civic Morals, Glencoe, Free Press, 1958.

DURKHEIM, E. (1968), Les règles de la méthode sociologique, $17^{\circledR}$ ed., Paris, PUF.

ERICKSON, K. (1966), Waymard Puritans, New York, John Wiley.

FLACKS, R. (1988), Making History, New York, Columbia University Press.

FOUCAULT, M. (1975), Surveiller et punir. Naissance de la prison, Paris, Gallimard,

GALliHER, J. F. et WALKER, A. (1977), «The Puzzle of the Social Origins of the Marihuana Tax Act of 1937», Social Problems, vol. 24, p. 367-376.

GARLAND, D. (1990), «Frameworks of Inquiry in the Sociology of Punishment ", British Journal of Sociology, vol. 41, p. 1-15.

GENET, J. (1949), Journal du voleur, Paris, Gallimard, 1982.

GRAMSCI, A. (1929-1935), Selections from the Prison Notebooks, New York, International Publishers, 1971.

GRAMSCI, A. (1926), «Some aspects of the Southern Question», p. 441-462, in A. GRAMSCI, Selections from Political Writings (1921-1926), New York, International Publishers, 1978.

GREenBERG, D. F. (1980), « Penal Sanctions in Poland: A Test of Alternative Models», Social Problems, vol. 28, p. 194-204.

GREENBERG, D. F. (1977), "The Dynamics of Oscillatory Punishment Processes *, The Journal of Criminal Law and Criminology, vol. 68, p. 643-651.

GUSFIELD, J. (1981), The culture of Public Problems. Drinking-driving and the Symbolic Order, Chicago, University of Chicago Press. 
HAY, D. (1975), «Property, Authority, and the Criminal Law», p. 17-63, in D. Hay, P. Linebaugh, J. G. Rule, E. P. Thompson et C. Winslow, Albion's Fatal Tree: Crime and Society in Eighteenth-Century England, New York, Pantheon Books.

HOBSBAWM, E. J. (1959), Primitive Rebels, New York, W. W. Norton, 1965.

INSOLERA, G. (1975), «La politica criminale nei discorsi dei Procuratori Generali (anno 1975)», La Questione Criminale, vol. 1, p. 289-317.

INVERARITY, J. et McCARTY, D. (1988), «Punishment and Social Structure Revisited: Unemployment and Imprisonment in the United States, 1948-1984», The Sociological Quarterly, vol. 29, p. 263-279.

JANKO VIC, I. (1977), «Labor Market and Imprisonment », Crime and Social Justice, vol. 8, p. 17-31.

KILLIAS, M. et GRANJEAN, C. (1986), «Chômage et taux d'incarcération: l'exemple de la Suisse de 1890 à 1941 », Déviance et Société, vol. 10, p. 309-322.

LAFFARGUE, B. et GODEFROY, T. (1989), «Economic Cycles and Punishment: Unemployment and Imprisonment (A time-series study: France, 1920-1985)", Contemporary Crises, vol. 13, p. 371-404.

LAMP, R. (1989), «Imprisonment and Economic Crisis", document non publié, Department of Sociology, University of California, Davis.

LINEBAUGH, P. (1976), « Karl Marx, the Theft of Wood, and Working Class Composition: A Contribution to the Current Debate», Crime and Social Justice, vol. 6, p. $5-16$.

MARX, K. (1852), «The Eighteenth Brumaire of Louis Bonaparte», p. 594-617, in R. C. Tucker (éd.), The Marx-Engels Reader, New York, W. W. Norton, 1978.

MARX, K. (1845), "The German Ideology", $1^{\text {tre }}$ partie, p. 146-200, in R. C. Tucker (éd.), The Marx-Engels Reader, New York, W. W. Norton, 1978.

MATZA, D. (1969), Becoming Deviant, Englewood Cliffs, N. J., Prentice-Hall.

MEAD, G. H. (1934), Mind, Self and Society, Chicago, The University of Chicago.

MEAD, G. H. (1917-1918), *The Psychology of Punitive Justice $»$, p. 212-239, in G. H. Mead, Selected Writings, Indianapolis, Bobbs-Merrill, 1964.

MELOSSI, D. (1990), The State of Social Control. A Sociological Study of Concepts of State and Social Control in the Making of Democracy, New York, St Martin's Press - Cambridge (UK), Polity Presso.

MELOSSI, D. (1985), «Punishment and Social Action: Changing Vocabularies of Punitive Motive Within A Political Business Cycle», Current Perspectives in Social Theory, vol. 6, p. 169-197.

MELOSSI, D. (1980), «Georg Rusche: A Biographical Essay», Crime and Social Justice,vol. 14, p. 51-63.

MELOSSI, D. (1978), «Georg Rusche and Otto Kirchheimer : Punishment and Social Structure ", Crime and Social Justice, vol. 9, p. 73-85. 
MELOSSI, D. (1976), * Institution of Social Control and Capitalist Organization of Work», p. 90-99 in B. Fine et al. (éd.), Capitalism and Rule of Law, Londres, Hutchington, 1979.

MILLS, C. W. (1956), The Power Elite, New York, Oxford University.

MILLS, C. W. (1940), «Situated Actions and Vocabularies of Motive», p. 439-452, in C. W. Mills, Power Politics and People, New York, Oxford University, 1963.

MILLS, C. W. (1939), "Language, Logic and Culture», p. 423-438, in C. W. Mills, Power Politics and People, New York, Oxford University, 1963.

RUSCHE, G. (1933), «Labor Market and Penal Sanction», Crime and Social Justice, vol. 10, p. 2-8.

RUSCHE, G. (1939), Punishment and Social Structure, New York, Russell \& Russell (1968).

SALVEMINI, G. (1963), Movimento Socialista e Questione Meridionale, Opere, vol. $4, \mathrm{n}^{\circ} 2$, Milan, Feltrinelli.

SHIBUTANI, T. (1962), «Reference Groups and Social Control», p. 128-147, in A. M. Rose (ed.), Human Behavior and Social Processes, Boston, Houghton Mifflin.

SPECTOR, M. et KITSUSE, J. (1977), Constructing Social Problems, Menlo Park, CA, Cummings.

SUDNOW, D. (1965), «Normal Crimes », Social Problems, vol. 12, p. 255-276.

WEBER, M. (1904-1905), The Protestant Ethic and the Spirit of Capitalism, New York, Scribner's, 1958.

YEAGER, M. G. (1979), « Unemployment and Imprisonment», The Journal of Criminal Law and Criminology, vol. 70, p. 586-588. 